\title{
Design and Capacity Analysis of Cellular-Type Underwater Acoustic Networks
}

\author{
Milica Stojanovic
}

\begin{abstract}
The design of a cellular underwater network is addressed from the viewpoint of determining the cell size and the frequency reuse pattern needed to support a desired number of users operating over a given area within a given system bandwidth. By taking into account the basic laws of underwater acoustic propagation, it is shown that unlike in the terrestrial radio systems, both the cell radius $R$ and the reuse number $N$ must satisfy a set of constraints to constitute an admissible solution (which sometimes may not exist). The region of admissible $(R, N)$, which defines the possible network topologies, is determined by the user density and the system bandwidth $(\rho, B)$, and by the required signal-to-interference ratio and per-user bandwidth $\left(\mathrm{SIR}_{0}, W_{0}\right)$. The system capacity is defined as the maximal user density $\rho_{\max }$ that can be supported within a given bandwidth, and it is derived analytically. Numerical examples are used to illustrate the results. It is shown that capacity-achieving architectures are characterized by $N$, which grows with $\rho_{\max }$. The capacity, as well as the range of admissible solutions, is heavily influenced by the choice of frequency region to which the bandwidth is allocated. Moving to a higher frequency region than that dictated by signal-to-noise ratio (SNR) maximization improves the SIR and yields a greater capacity. Although higher frequencies demand greater transmission power to span the same distance, they also imply a reduction in the cell size, which, in turn, provides an overall reduction in the transmission power. While complex relationships are involved in system optimization, the analysis presented offers a simple tool for the design of future ocean observation systems based on cellular types of network architecture for wide area coverage.
\end{abstract}

Index Terms-Cellular systems, network capacity, resource allocation, spatial reuse, underwater acoustic networks.

\section{INTRODUCTION}

W ITH the maturing of underwater acoustic modem technology, the concept of an underwater network of distributed autonomous nodes is coming closer to realization. The first experimental deployments are underway, whose goal is to demonstrate the existing hardware capabilities [1], [2]. At the same time, research is gaining momentum on the design of multiple-access methods [3], [4] and medium access control protocols [5]-[9] for underwater acoustic systems, and beginning to address the issues of routing. However, the fundamental questions of network topology optimization and the associated capacity analysis remain unaddressed [10].

Manuscript received March 23, 2007; accepted February 12, 2008. First published October 10, 2008; current version published October 31, 2008. This work was supported by the National Science Foundation under Grants 0427502, 0532223, and 0520075.

Associate Editor: J. Preisig.

The author is with the Massachusetts Institute of Technology (MIT), Cambridge, MA 02139 USA (e-mail: millitsa@mit.edu).

Color versions of one or more of the figures in this paper are available online at http://ieeexplore.ieee.org.

Digital Object Identifier 10.1109/JOE.2008.920210
The design of underwater networks is daunted by the constraints of acoustic propagation: limited, distance-dependent bandwidth, poor quality of the physical link, and high channel latency caused by the low speed of sound all conspire to produce a communication medium of extreme difficulty, which poses challenging research problems. The harshness of the environment and the associated deployment difficulties, as well as the size and cost of underwater nodes, make the underwater acoustic networks very different from their land-based radio counterparts, preventing a direct application of the existing principles and requiring a dedicated system design [11].

The development of future underwater acoustic networks can be envisioned in two extreme directions: centralized networks, in which the distributed nodes rely on an infrastructure of base stations through which to communicate, and decentralized networks in which there is no a priori established infrastructure, but the nodes alone are responsible for multihop message relaying. While the first type of architecture follows the paradigm of a cellular system, the second type follows the paradigm of an ad hoc network. The two concepts are illustrated in Fig. 1. Naturally, hybrid architectures are also likely to emerge, driven by particular system requirements and applications.

In this paper, we focus on the cellular type of underwater networks, addressing top-level system design and its implications on the system capacity. As an example application, one may think of a fleet of autonomous vehicles, deployed on a collaborative mission to map a wide area of the ocean floor. The base stations in such a system may be mounted on the surface buoys (radio-based infrastructure) or on the bottom (cable-based infrastructure). ${ }^{1}$ It is also possible to assign the roles of base stations to a set of distributed nodes, and establish an "infrastructure" through a separate acoustic channel, perhaps one that uses a lower frequency band to span longer distances.

Cellular networks have revolutionized terrestrial mobile communications by introducing the concept of spatial frequency reuse. The simple idea of using the same frequency band in two cells that are sufficiently far apart so that they do not cause interference to each other enables spatial reuse of bandwidth, which, in turn, ensures coverage of a large area within the practical constraints of finite bandwidth. Since bandwidth is at premium in an acoustic channel, ${ }^{2}$ the concept of frequency reuse is an appealing one, and, given the immense success and practicality of terrestrial cellular systems, the question naturally arises as to

\footnotetext{
${ }^{1}$ Note that one could use both surface and bottom stations to provide full volume coverage in an underwater network. For simplicity, we restrict our attention to a 2-D problem.

${ }^{2}$ Acoustic bandwidth is not (yet) limited by frequency regulations, but by the fundamental nature of propagation.
} 

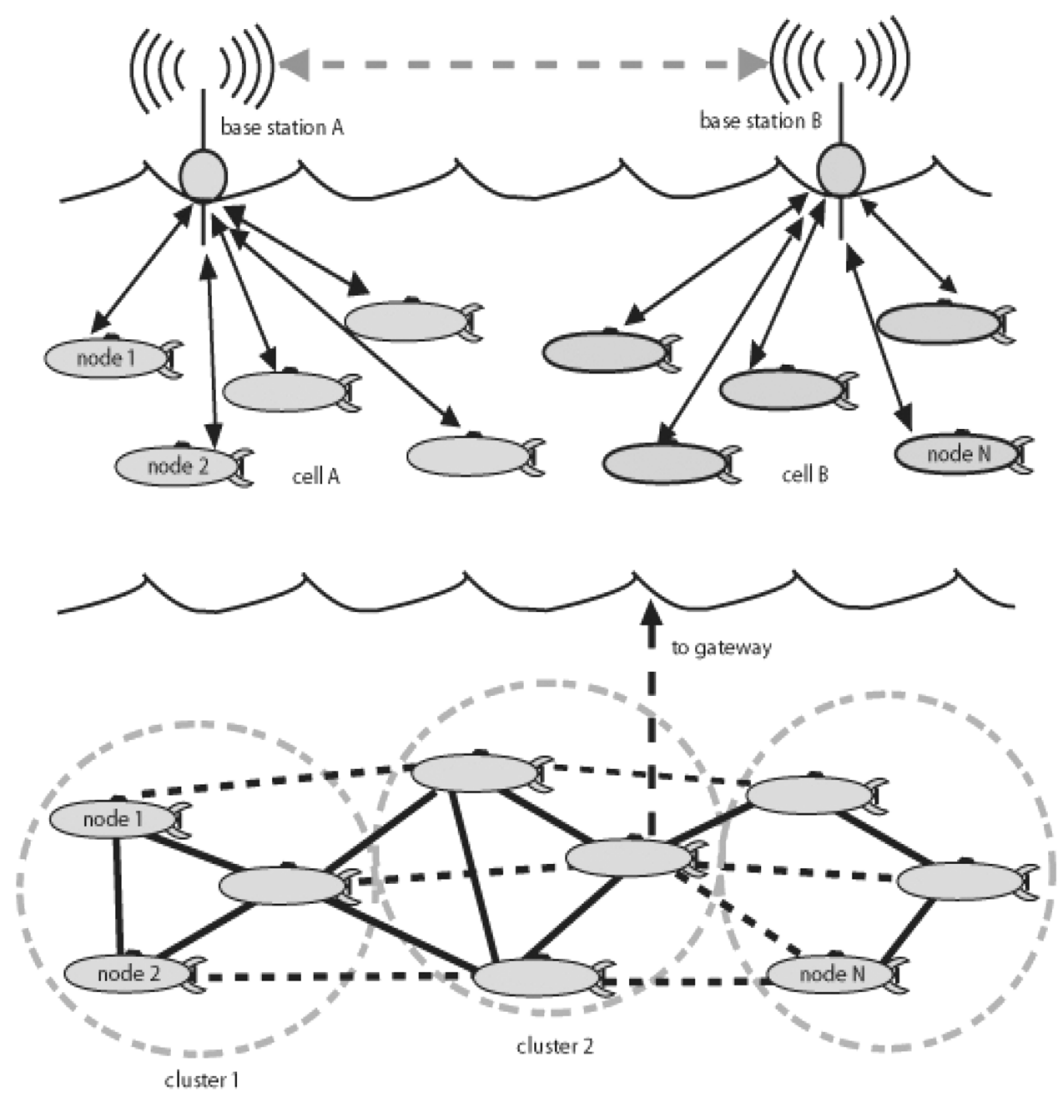

Fig. 1. Two possible types of future underwater networks: a cellular architecture is based on a separate infrastructure that links the central stations, while an ad hoc architecture is not. These types represent two extreme ends, between which many combinations are possible. For example, nodes in an ad hoc system may form clusters for better utilization of system resources (power and bandwidth) with the role of base station assigned to a cluster head [4].

how does the cellular concept apply to an underwater acoustic environment.

The first task in the design of a cellular network is the selection of network topology, i.e., determination of the cell size (radius) and the reuse pattern. In other words, the practical questions that one wants to answer are the following: For a given distribution of users and the desired information throughput, what should be the coverage area of one base station? What should be the distance to another base station operating in the same frequency band? According to what pattern should the frequency bands be reused?

In a terrestrial radio environment, this problem has a very simple solution that leads to the well-known reuse pattern of 7 , and selection of the smallest practical cell radius [12]. In an underwater acoustic environment, however, the solution is complicated by the fact that the path loss does not consist only of the spreading loss (which grows with distance as $d^{k}$, where $k$ is usually a number between 1 and 2), but the absorption loss as well [which grows with distance as $a^{d}(f)$, and depends on the frequency through the factor $a(f)]$. Because of this fundamental difference, simple principles of cellular radio system design do not apply to an underwater acoustic environment. Instead, complex relationships between the system parameters exist, which lead to a set of constrained solutions, and, sometimes, to a situation in which it is not possible to use the cellular concept at all.

The ultimate question that one wants to answer is that of the system capacity: What is the maximal number of users that can be supported over a certain area of coverage within a given bandwidth? Currently, no analytical results exist that can be used even as rough guidelines to estimate this number.

In this paper, we address the questions raised previously. In Section II, the problem of underwater cellular system design is formulated, and the admissible solutions are outlined. Capacity analysis is presented in Section III, along with numerical illustrations and discussion of results. Section IV is devoted to the issues of power and bandwidth allocation. Finally, conclusions are summarized in Section V.

\section{SYSTEM DESIGN}

We consider a system layout that follows the basic cellular concept of spatial frequency reuse that comes with time-division multiple access (TDMA) or frequency-division multiple access (FDMA) [12]. This concept is illustrated in Fig. 2.

Specifically, we address the following scenario: given a density of users $\rho$ (in users per squared kilometer) and a total system bandwidth $B$ (in hertz), we want to find the cell radius $R$ (in meters) and the frequency reuse number $N$, such that the number of users per hertz of occupied bandwidth is maximized, subject to the fact that the following conditions are met.

1) The cochannel signal-to-interference ratio (SIR) remains greater than a predetermined level SIR $\geq \mathrm{SIR}_{0}$.

2) The bandwidth (throughput) per user remains greater than a prespecified value $W \geq W_{0}$. 


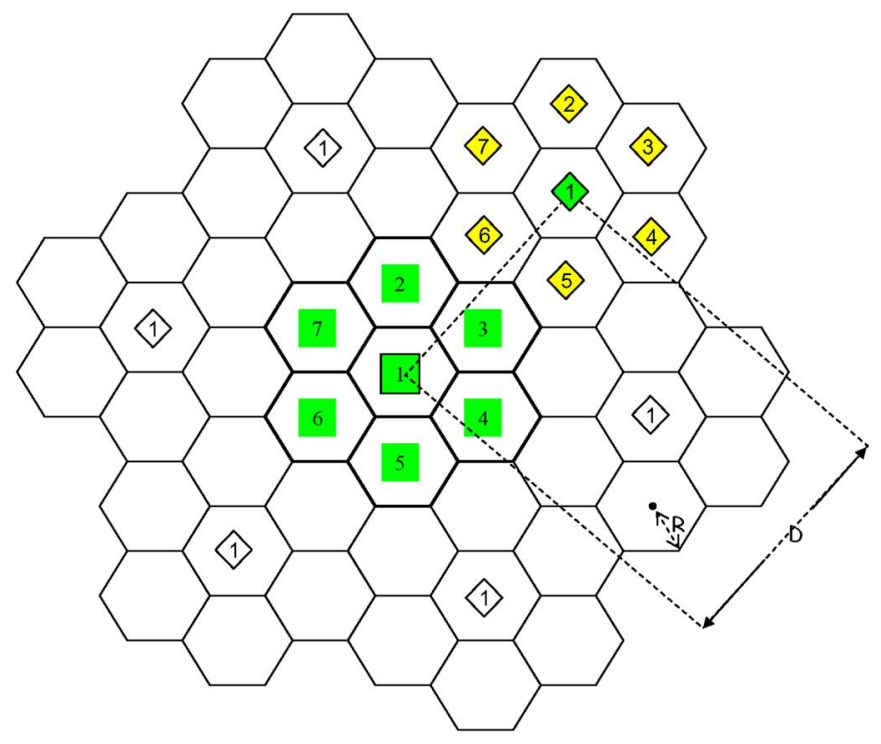

Fig. 2. Total bandwidth $B$ is divided among $N$ adjacent cells (seven in this example), which form a cluster. The clusters are then replicated over an arbitrary area. The cells marked by the same number operate in the same frequency band, but they are far apart from each other. The cell radius is $R$, and the distance between two cochannel cells (the frequency reuse distance) is $D$.

\section{A. Signal-to-Interference Ratio}

We start by evaluating the cochannel SIR, taking into account the acoustic propagation loss. Assuming a hexagonal cell geometry, as illustrated in Fig. 2, the cochannel interference has as its dominant component the signals coming from the nearest six cochannel cells. The least favorable conditions occur at the cell edge, where the SIR is approximately defined by the ratio of powers

$$
\operatorname{SIR}=\frac{P(R)}{6 P(D)}
$$

Here, $R$ is the distance traveled by the desired signal and $D$ is the distance traveled by the interfering signal. In a 2-D system with hexagonal cells, the two distances are related by $D=Q R$, where $Q=\sqrt{3 N}$ is the reuse factor. The reuse number $N$ cannot be chosen arbitrarily; instead, it is given in a form $N=$ $i^{2}+i j+j^{2}$, where $i$ and $j$ are nonnegative integers [12]. This choice makes it possible to replicate the clusters (the patterns of $N$ hexagons) across an arbitrary area in a uniform manner, i.e., to perform tessellation.

In a radio system, the signal power attenuates with distance as $P(d) \sim 1 / d^{k}$, where $k$ is usually a number greater than 2 (the value for free line-of-sight propagation). The SIR is thus rendered independent of the cell radius $\operatorname{SIR}=Q^{k} / 6$ and the reuse number $N$ is easily determined from it. Its typical value in cellular radio systems is $N=7$, which ensures SIR greater than $17 \mathrm{~dB}$ for the two-ray ground reflection model with path loss exponent $k=4$.

In an acoustic channel, the simple path loss model does not hold. The acoustic path loss, experienced by a signal of frequency $f$ traveling over a distance $d$, is given by

$$
A(d, f)=A_{0} d^{k} a^{d}(f)
$$

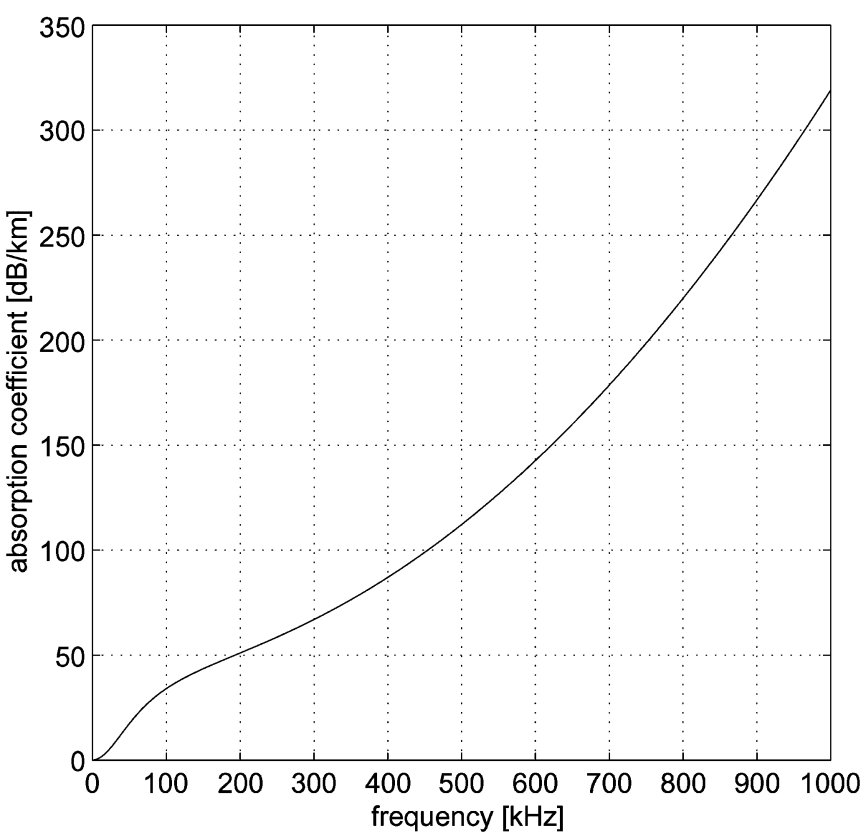

Fig. 3. Absorption coefficient $a(f)$ is given in decibels per kilometer as $10 \log a(f)=0.11 f^{2} /\left(1+f^{2}\right)+44 f^{2} /(4100+f)+2.75 \cdot 10^{-4} f^{2}+0.003$, where $f$ is the signal frequency in kilohertz. This empirical formula is valid for frequencies greater than a few hundred hertz.

where $A_{0}$ is a normalizing constant, $k$ is the spreading factor (the values 1 and 2 correspond to cylindrical and spherical spreading, respectively), and $a(f)$ is the absorption coefficient. Without the loss of generality, we will assume in the later numerical examples a practical spreading factor $k=1.5$ and absorption according to Thorp [13], which is reproduced in Fig. 3.

The signal power at a distance $d$ from the transmitter is now evaluated as

$$
P(d)=\int_{f_{n}}^{f_{n}+B_{0}} S(f) A^{-1}(d, f) d f
$$

where $S(f)=P_{T} / B_{0}$ is the power spectral density of the transmitted signal, which we assume to be flat (and as such not relevant for the SIR), and the integration is carried over the frequency band occupied by the signal, starting at some $f_{n}$ and extending over a bandwidth $B_{0}$.

The signal bandwidth $B_{0}$ depends on the multiple-access technique used. If TDMA is used, it equals the bandwidth allocated to one cell, $B_{0}=B / N$. If FDMA is used, it equals the width of a frequency channel allocated to one of $U$ users sharing a cell, $B_{0}=(B / N) / U$. In what follows, we will assume a TDMA system, keeping in mind that the analysis can easily be modified to accommodate FDMA, and, hence, there is no loss of generality.

The frequency $f_{n}$ differs among the $N$ cells, and, due to the frequency dependence of the acoustic path loss, different cells experience different attenuation. In particular, higher bands experience greater attenuation. However, this is true both for the desired signal and for the interfering signals, with the overall effect that the SIR improves with an increase in frequency. Hence, 


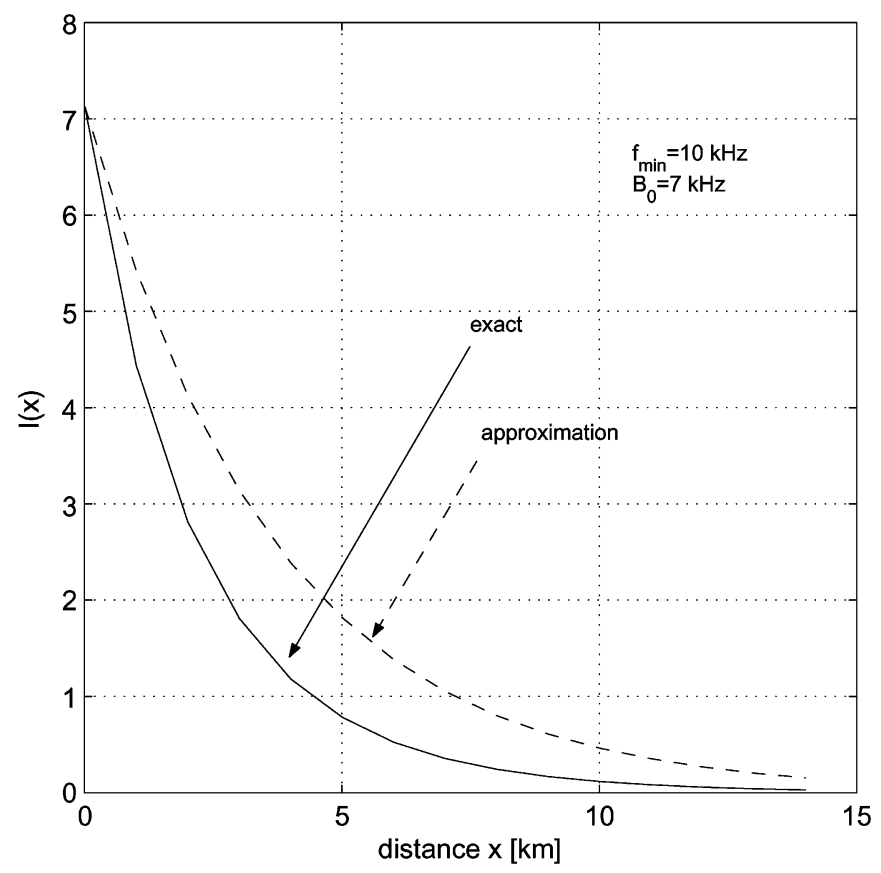

Fig. 4. $I(x)$ as defined in (5) and its approximation (6).

to ensure that the worst case conditions are met, the system design should be carried out for the lowest frequency band, which is the one at the band edge $f_{1}=f_{\min }$. By extension, the design of an FDMA system should be carried out for the lowest frequency channel of the lowest frequency band.

Using the acoustic path loss to determine the received powers, the SIR condition can be expressed in terms of the cell radius $R$ and the reuse factor $Q$ as

$$
\mathrm{SIR}=\frac{1}{6} Q^{k} \frac{I(R)}{I(Q R)} \geq \operatorname{SIR}_{0}
$$

where

$$
I(x)=\int_{f_{\min }}^{f_{\min }+B_{0}} a^{-x}(f) d f .
$$

Hence, the SIR in an acoustic system depends both on the reuse number and the cell radius through the terms $I(R)$ and $I(Q R)$. An explicit dependence of the SIR on $N$ and $R$ could be obtained if one were to approximate the attenuation as frequency independent, $a(f) \approx a\left(f_{\min }\right)=a_{0}$. Then, we would have

$$
I(x) \approx a_{0}^{-x} B_{0}
$$

and

$$
\operatorname{SIR} \approx \frac{1}{6} Q^{k} a_{0}^{(Q-1) R} .
$$

At this point, we may note the difference between an acoustic and a radio system: the latter can be viewed as a special case with $a_{0}=1$, yielding an SIR that is independent of the cell radius. Moreover, the approximation is acceptable only for $B_{0} \ll f_{\min }$, which is rarely the case in an acoustic system. Fig. 4 shows the function $I(x)$ and its approximation (6) for an arbitrary selection of the system parameters: $f_{\min }=10 \mathrm{kHz}$ and $B_{0}=7 \mathrm{kHz}$ (the latter would correspond to a total bandwidth $B$ of about $50 \mathrm{kHz}$ and $N=7$ in a TDMA system).

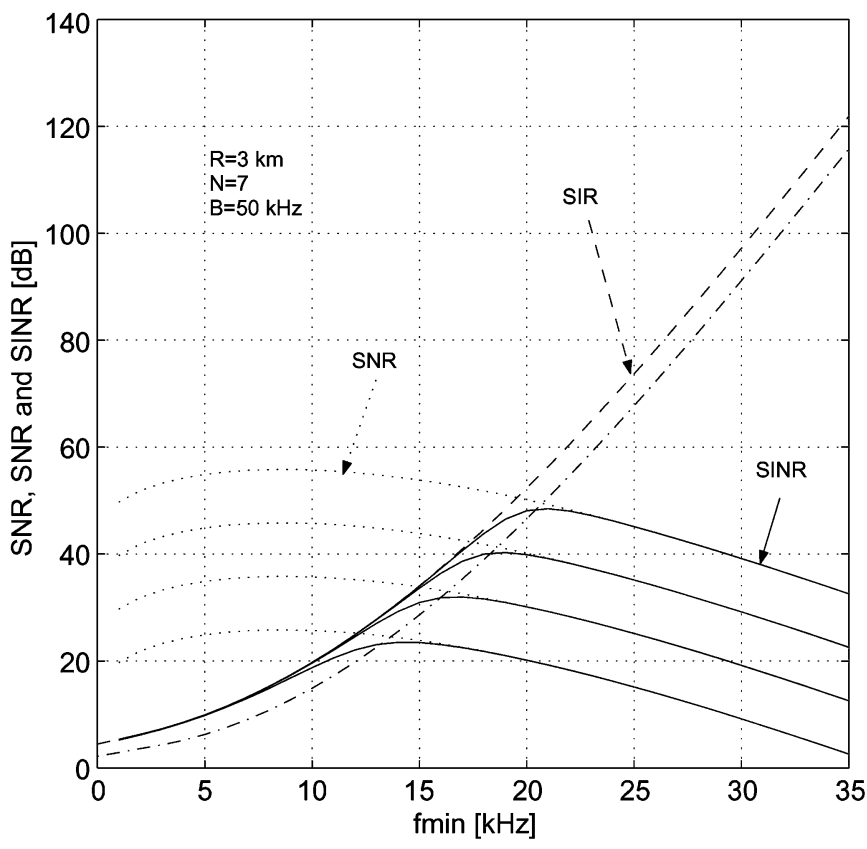

Fig. 5. SNR, SIR, and SINR versus $f_{\text {min }}$. The approximation (7) for the SIR is shown in dash-dot line below the actual SIR. Several transmission power levels are used (ranging from 120 to $150 \mathrm{~dB}$ re $1 \mu \mathrm{Pa} @ 1 \mathrm{~m}$ in steps of 10 $\mathrm{dB})$ : changing the transmission power affects the SNR, but not the SIR. Noise power is calculated using ambient noise power spectral density corresponding to moderate shipping activity and no wind, as in [10].

In addition to being dependent on the cell radius and the reuse number, the SIR also depends on the band-edge frequency $f_{\min }$, and, to a lesser extent, on the bandwidth $B_{0}$. Fig. 5 illustrates the SIR as a function of $f_{\min }$. The SIR increases with frequency because $a(f) \geq 1 \forall f$. Using a higher frequency band thus ensures a greater SIR; however, it results in higher attenuation, making the signal more vulnerable to noise. The signal-to-noise ratio (SNR) is also illustrated in the figure, along with the resulting signal-to-noise-plus-interference ratio (SINR). A practical system is normally designed such that the noise is negligible with respect to interference, i.e., SNR $\gg S I R$, or, equivalently, SINR $\approx$ SIR. Hence, SIR is used as a figure of merit for the system design.

Selection of the optimal $f_{\min }$ is obviously influenced by many factors, including the physical constraints of the transducers and the power budget. For the moment, we lay these large scale system design issues aside, and focus on the selection of the cell radius and the reuse pattern, assuming that $f_{\min }$ is set a priori. In the numerical examples that follow, we will use $f_{\min }=10 \mathrm{kHz}$.

\section{B. Cell Radius and the Reuse Number}

Fig. 6 shows the SIR as a function of the cell radius for different values of the reuse number. The SIR increases with both $R$ and $N$. Thus, in order for the SIR to be greater than the design value $\mathrm{SIR}_{0}$, the cell radius has to be greater than some minimum, which we express as

$$
R \geq R_{0}(N)
$$

The minimal cell radius depends on the reuse pattern $N$, and also on the required SIR and the system bandwidth. There is no 


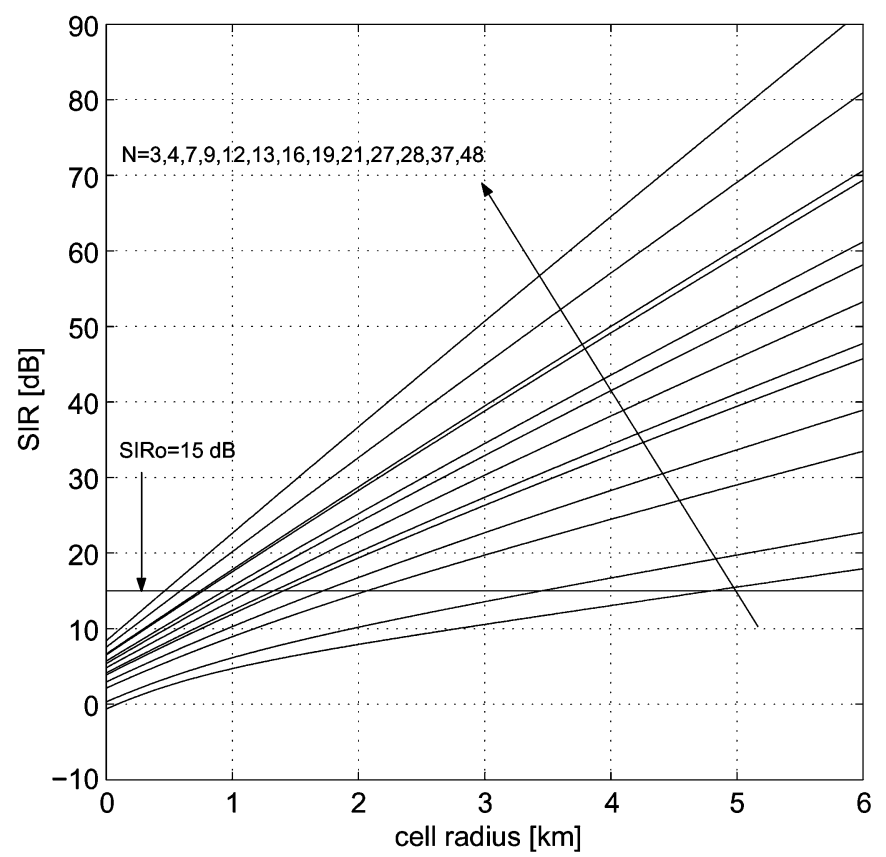

Fig. 6. SIR as a function of the cell radius $R$ for different values of the reuse number $N$.

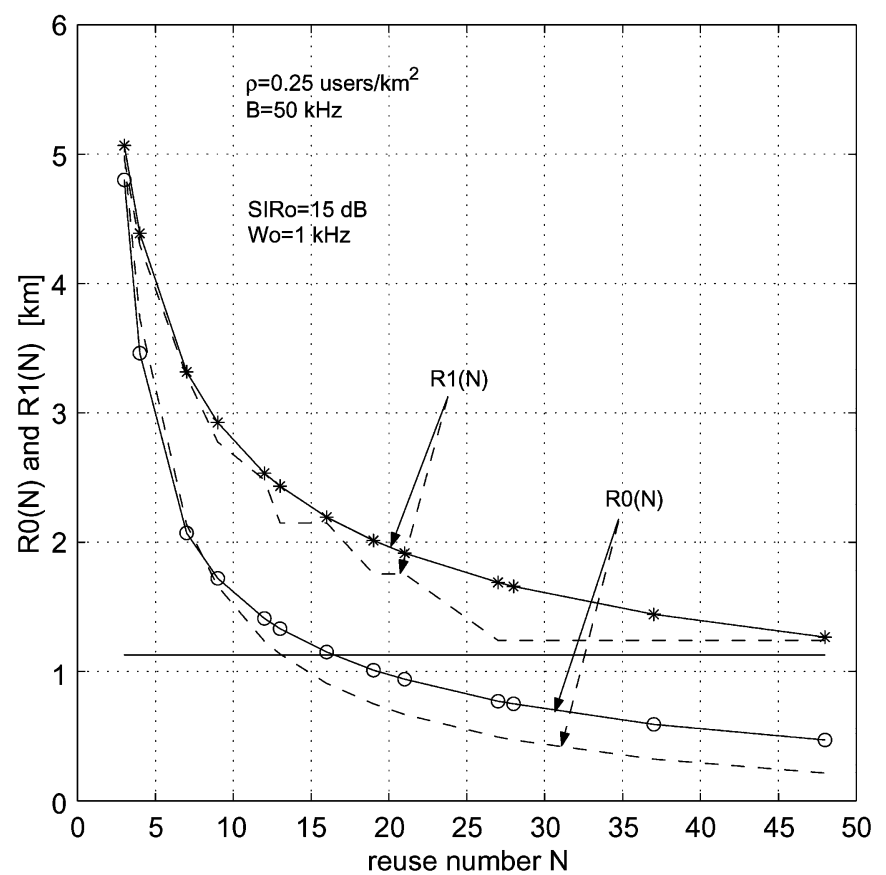

Fig. 7. Region of acceptable values $(R, N)$ is defined by (14): it is bounded by $R_{0}(N), R_{1}(N)$, and the straight line which lies at $1 / \sqrt{\alpha \rho}$. Shown in dashed line are the approximation (9) for $R_{0}(N)$, and $R_{1}(N)$ as defined in (12).

closed-form expression for $R_{0}(N)$, unless the approximation (6) is used, which would yield

$$
R_{0}(N) \approx \frac{10 \log \operatorname{SIR}_{0}+4-k 10 \log Q}{(Q-1) 10 \log a_{0}} .
$$

Again, this approximation should be used only for illustrative purposes, to gain insight into the rate of decay of the minimal radius with $N$. The exact function $R_{0}(N)$ is shown in Fig. 7, which we will discuss shortly.
First, let us analyze the second system constraint, namely, the requirement that the per-user bandwidth be $W \geq W_{0}$. For a given density of users $\rho$, the number of users per cell is $\rho \alpha R^{2}$, where $\alpha=3 \sqrt{3} / 2$ for the hexagonal cell geometry $(\alpha=\pi$ if the cells are modeled as circular). The bandwidth allocated to one cell is $B / N$, and, hence, the bandwidth per user must satisfy

$$
W=\frac{B / N}{\rho \alpha R^{2}} \geq W_{0}
$$

In order for this condition to hold, the cell radius has to be less than some maximum, which we express as

$$
R \leq R_{1}(N)=\frac{1}{\sqrt{\alpha \rho}} \sqrt{\frac{B}{N W_{0}}} .
$$

One may also want to specify the number of users per cell as an integer, in which case the condition (10) should be restated to reflect the worst case. From the viewpoint of per-user bandwidth, the worst case occurs when the number of users per cell is the greatest. Given that this number is an integer, condition (10) implies that it can be at most $\left\lfloor B / N W_{0}\right\rfloor$. The corresponding maximal cell radius is

$$
R_{1}(N)=\frac{1}{\sqrt{\alpha \rho}} \sqrt{\left\lfloor\frac{B}{N W_{0}}\right\rfloor} .
$$

The difference between the two expressions for $R_{1}(N)$ is not a fundamental one, and in what follows, we will use the definition (11) without the loss of generality.

Finally, the number of users in a cell should be greater than one, as the cellular concept is otherwise meaningless. This fact yields an additional condition

$$
R \geq \frac{1}{\sqrt{\alpha \rho}}
$$

Combining the requirements (8), (11), and (13), we find that the cell radius must satisfy

$$
\bar{R}_{0}(N)=\max \left\{R_{0}(N), \frac{1}{\sqrt{\alpha \rho}}\right\} \leq R \leq R_{1}(N) .
$$

This expression defines the admissible region of $(R, N)$. Only those values of $(R, N)$ that belong to this region constitute a valid design.

Fig. 7 illustrates the admissible region for a system with $\rho=$ $0.25 \mathrm{users} / \mathrm{km}^{2}$ (1 user per $2 \mathrm{~km} \times 2 \mathrm{~km}$ ), $B=50 \mathrm{kHz}, \mathrm{SIR}_{0}=$ $15 \mathrm{~dB}$, and $W_{0}=1 \mathrm{kHz}$. This region is bounded by $R_{0}(N)$, $R_{1}(N)$, and the straight line $1 / \sqrt{\alpha \rho}$. Markers are placed on the curves to indicate possible values of $N(3,4,7$, etc.).

We observe that $R_{0}(N)$ decays faster than $R_{1}(N)$. The smallest value of $N$ for which $R_{0}(N)$ falls below $R_{1}(N)$ defines the lowest reuse pattern that can be used. The point at which $R_{1}(N)$ falls below $1 / \sqrt{\alpha \rho}$ determines the maximal reuse number. For each admissible value of $N$, there is a range of cell radii that can be chosen to design the system. In practice, it is desirable to use a small value of $N$ because it facilitates the frequency allocation process and minimizes the loss incurred by insertion of the necessary guard bands. In this example, $N$ can be chosen as low as 3 . However, we may want to choose $N$ somewhat greater than the minimum to ensure a margin for the selection of the cell radius. 


\section{Number of Users and Bandwidth per User}

Once the reuse number is fixed, the cell radius $R$ can be chosen as any value between the minimum $\bar{R}_{0}(N)$ and the maximum $R_{1}(N)$. The selection depends on the system optimization criterion. A natural criterion to consider is maximization of the number of users supported per hertz of occupied bandwidth

$$
C=\frac{\rho \alpha R^{2}}{B / N} .
$$

To maximize this quantity for a given $N$, while respecting the constraints $\mathrm{SIR} \geq \mathrm{SIR}_{0}$ and $W \geq W_{0}$, the maximum value of the cell radius should be chosen, $R=R_{1}(N)$. This selection results in the maximum $C_{\max }=1 / W_{0}$ and favors SIR over the bandwidth that can be allocated to each user. Whenever the cell radius is chosen greater than $R_{0}(N)$, the SIR will be greater than the design value $\mathrm{SIR}_{0}$; when $R=R_{1}(N)$, the SIR will equal some $\operatorname{SIR}_{\max }(N)$.

Alternatively, it may be desired that the per-user bandwidth $W$ be maximized. In that case, the smallest cell radius should be chosen, $R=\bar{R}_{0}(N)$. This selection yields the maximum

$$
W_{\max }(N)=\frac{B / N}{\rho \alpha \bar{R}_{0}^{2}(N)} .
$$

The corresponding number of users per hertz of occupied bandwidth is $C_{\min }(N)=1 / W_{\max }(N)$.

Hence, depending upon the chosen $N$, the actual available bandwidth per user $W$, the SIR, the number of users per hertz of occupied bandwidth $C$, and the associated number of users per cell $U=\rho \alpha R^{2}$ depend on the cell radius and have values that lie between some minima and maxima. Fig. 8 illustrates the bounds on these quantities.

\section{Sensitivity to System Parameters and Performance Requirements}

In the example considered, there is a large range of admissible $(R, N)$. However, a slight change in the system requirements $\left(\operatorname{SIR}_{0}, W_{0}\right)$ and/or system parameters $(\rho, B)$ may lead to a situation in which the range of solutions narrows, and also to a situation in which there is no solution. These situations are illustrated in Fig. 9.

An increase in the required $\mathrm{SIR}_{0}$ causes $R_{0}(N)$ to increase, while an increase in the required $W_{0}$ causes $R_{1}(N)$ to decrease. Hence, increasing the requirements on the system performance $\left(\operatorname{SIR}_{0}, W_{0}\right)$ causes the region of admissible $(R, N)$ to tighten. This fact is illustrated in Fig. 9(a), which shows the admissible region $(R, N)$ for the same system parameters $(\rho, B)$ as before, but stricter requirements, $\operatorname{SIR}_{0}=17 \mathrm{~dB}$ and $W_{0}=1.5 \mathrm{kHz}$. Note that while $R_{0}(N)$ is governed by $\operatorname{SIR}_{0}$, and $R_{1}(N)$ by $W_{0}$, both radii depend on the bandwidth $B$. Fig. 9(b) shows an example when there is no solution that can accommodate the required $\left(\operatorname{SIR}_{0}, W_{0}\right)$ for the specified $(\rho, B)$. In such a situation, it is not possible to employ a cellular system architecture to design the network.

We must also keep in mind that $R_{0}(N)$ depends on the bandedge frequency $f_{\min }$. Fig. 10 illustrates this dependence. We observe that $R_{0}(N)$ is heavily influenced by $f_{\min }$. Moving to higher frequencies improves the SIR (recall Fig. 5), which in turn allows $R_{0}(N)$ to be reduced, effectively opening up the range of admissible solutions. For example, if we consider the same system parameters as those of Fig. 7, but increase the user density to $\rho=5$ users $/ \mathrm{km}^{2}$ (an increase by a factor of $20 \mathrm{com}$ pared to $\rho=0.25$ users $/ \mathrm{km}^{2}$ ), there is no solution with $f_{\min }=$ $10 \mathrm{kHz}$. However, if the operational frequency range is moved to $f_{\min }=20 \mathrm{kHz}$, the system can be designed with $N$ as low as 7 .

\section{CAPACITY ANALYSIS}

Our discussion thus far indicates that complex relationships govern the design of a cellular system for the underwater acoustic environment. The fact that the range of admissible network topologies is determined by the desired user density (among other parameters) gives rise to the question of user capacity. We define the capacity of an underwater acoustic cellular network as the maximal user density that can be supported within a given bandwidth.

To assess the user capacity, we turn to the design condition (14). This condition implies that in order for a valid design to exist, the following inequality must hold:

$$
\bar{R}_{0}(N) \leq R_{1}(N) .
$$

Substituting for $\bar{R}_{0}(N)$ and $R_{1}(N)$, this condition can be expressed in terms of the user density and the system bandwidth. To do so, we observe that for any given $N$, there are two possibilities: 1) $\rho \geq 1 / \alpha R_{0}^{2}(N)$ or 2) $\rho<1 / \alpha R_{0}^{2}(N)$. Note that $R_{0}(N)$ depends on the bandwidth $B$, and, hence, the possibilities 1$)$ and 2) correspond to some regions in the $(\rho, B)$ space.

If 1) is true, then in order for a solution to exist, condition (17) implies that the user density has to be $\rho \leq\left(B / W_{0}\right) / \alpha N R_{0}^{2}(N)$. Thus, the maximal density for a given $N$ is $\rho_{\max }(N)=\left(B / W_{0}\right) / \alpha N R_{0}^{2}(N)$ in case 1). The maximal density depends on the bandwidth explicitly, and also through $R_{0}(N)$.

If 2) is true, then in order for a solution to exist, the bandwidth has to be $B \geq N W_{0}$. Conversely, if $B<N W_{0}$, there is no solution, i.e., $\rho_{\max }(N)=0$.

Combining the two cases 1) and 2), we obtain the maximal user density that can be supported for a given $N$

$$
\rho_{\max }(N)=\left\{\begin{array}{ll}
\left(B / W_{0}\right) / \alpha N R_{0}^{2}(N), & B / W_{0} \geq N \\
0, & B / W_{0}<N
\end{array} .\right.
$$

Fig. 11 shows $\rho_{\max }(N)$ as a function of $N$, for several values of the bandwidth $B$. The maximal density, conditioned on a fixed reuse number, increases with $N$ until it reaches a point where $N$ exceeds $B / W_{0}$, after which it drops to zero. As the bandwidth increases, so does the conditional capacity $\rho_{\max }(N)$, as well as the range of reuse numbers that can be employed in a cellular architecture.

We now define the system capacity as the maximum of conditional capacities $\rho_{\max }(N)$, taken over the reuse number $N$

$$
\rho_{\max }=\max _{N} \rho_{\max }(N) .
$$

The capacity is a function of the system bandwidth $B$, and it also depends on the system requirements $\left(\operatorname{SIR}_{0}, W_{0}\right)$, as well as on the band-edge frequency $f_{\min }$. 

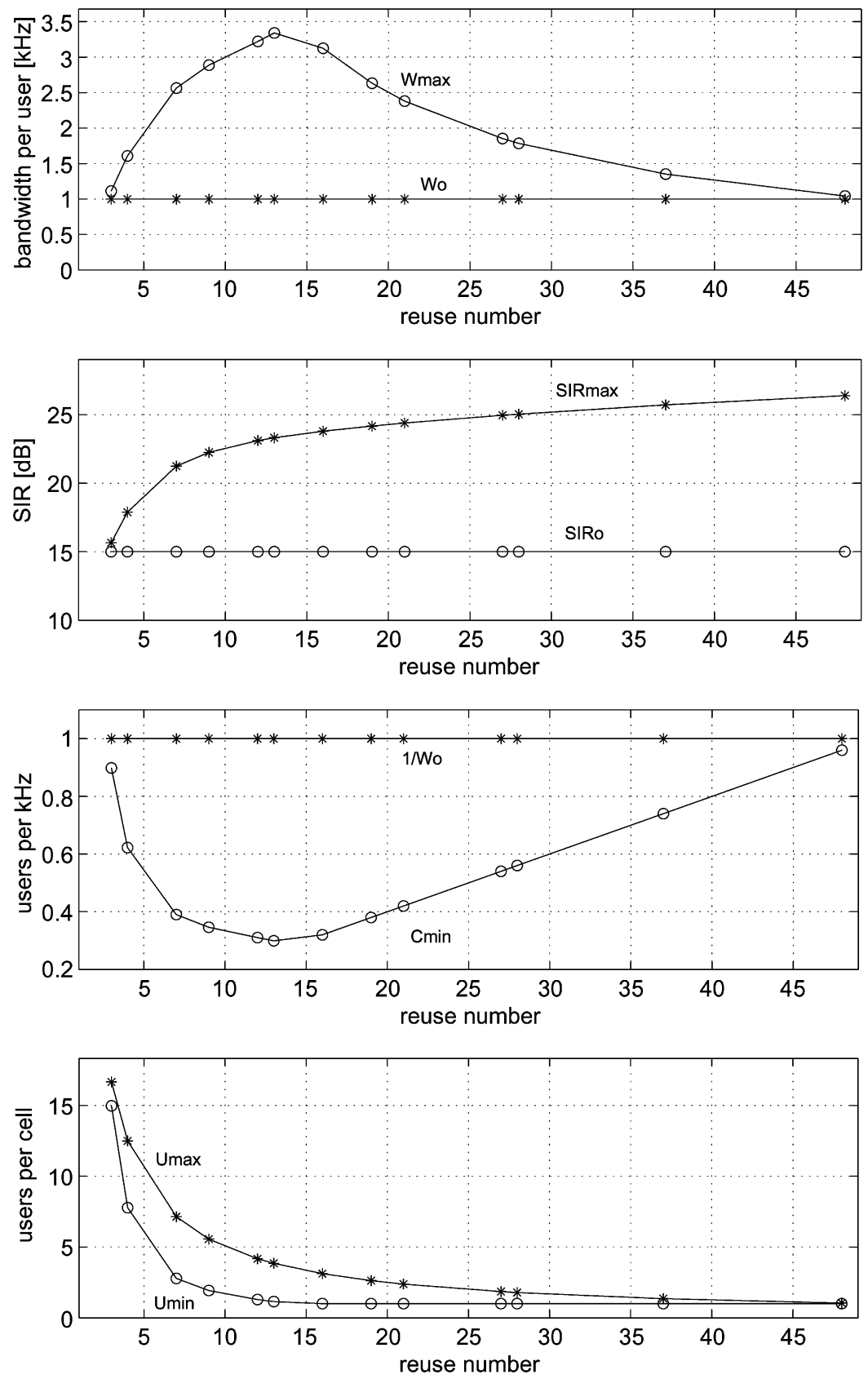

Fig. 8. Bounds on the available bandwidth per user $W$, number of users per hertz of occupied bandwidth $C$, SIR, and the number of users per cell $U$, all as functions of the reuse number $N$. Depending upon the cell radius, these quantities assume values between the minima and the maxima shown. Curves labeled with circles correspond to the selection of the cell radius as $R=\bar{R}_{0}(N)$, and those labeled with stars correspond to $R=R_{1}(N)$. System parameters are the same as those for Fig. 7: $\rho=0.25$ users $/ \mathrm{km}^{2}, B=50 \mathrm{kHz}, \mathrm{SIR}_{0}=15 \mathrm{~dB}$, and $W_{0}=1 \mathrm{kHz}$.

Fig. 12 illustrates the capacity for the system requirements used in the example of Fig. 7. Shown in dashed line is the set of maximal user densities $\rho_{\max }(N)$, for $N$ varying from 3 to 37 . The overall system capacity (19) is shown in solid line. Below this curve lies the region of points $(\rho, B)$ for which a cellular system can be designed.

The capacity increases with bandwidth, following a double trend: a sharp increase occurs at points $B / W_{0}=N$, which correspond to the valid reuse numbers $3,4,7$, etc., while between these points, the capacity curve follows the function $\rho_{\max }(N)$. For example, between $B / W_{0}=28$ and 37 , the capacity equals $\rho_{\max }(28)$ (highlighted dashed curve). The number $N=28$ is indicated on the capacity curve in this region, where it represents the reuse number needed to achieve the capacity, i.e., to support the associated density $\rho_{\max }$. The capacity increases abruptly to $\rho_{\max }(37)$ at $B / W_{0}=37$.

A key observation to be made from Fig. 12 is that the capacity-achieving architectures are characterized by the reuse number $N$ that grows with $\rho_{\max }$. Depending upon the system constraints and the desired performance, this fact may imply the need for a large $N$. To be specific, if we want to support a user density of 0.25 users $/ \mathrm{km}^{2}$, Fig. 12 indicates that a bandwidth of at least $19 \mathrm{kHz}$ is needed to meet the given system requirements. Using the minimal bandwidth will require $N=19$, which is a 


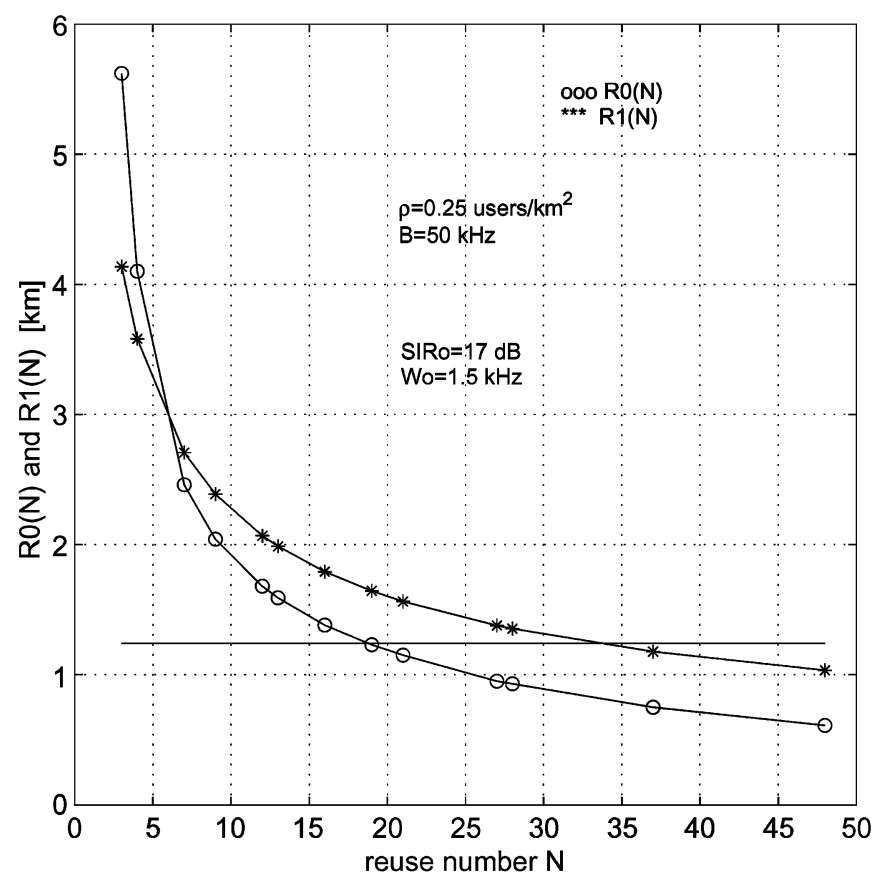

(a)

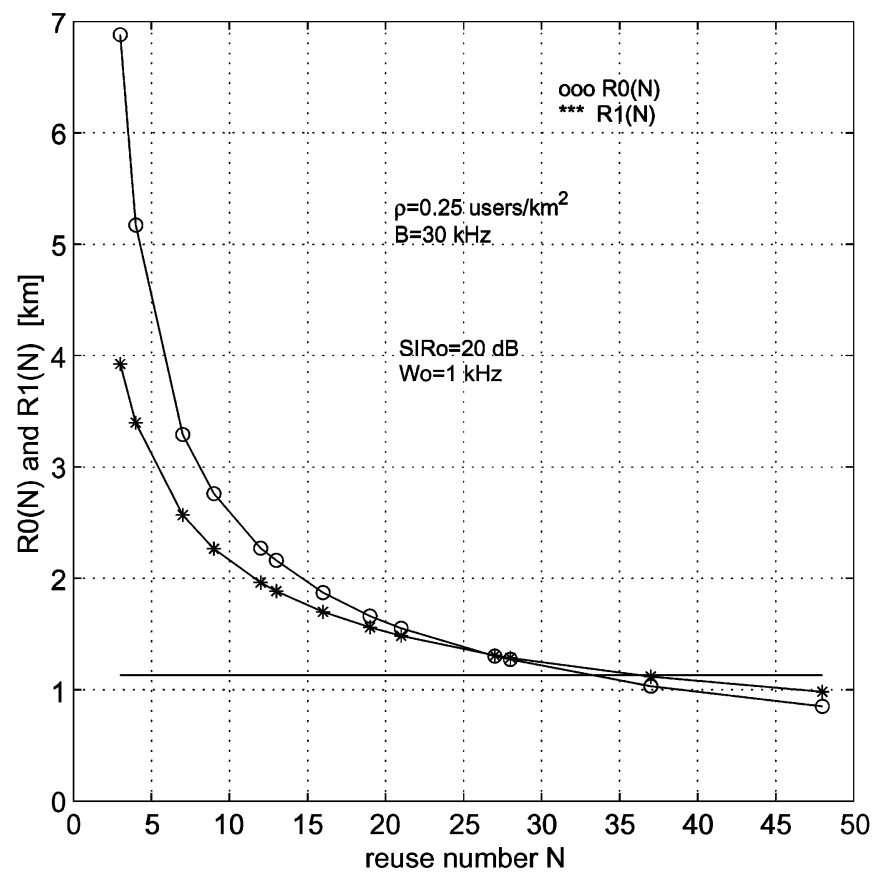

(b)

Fig. 9. (a) Region of acceptable $(R, N)$ narrows for stricter system requirements $\left(\mathrm{SIR}_{0}, W_{0}\right)$ as compared to those of Fig. 7. (b) There are no solutions $(R, N)$ that can accommodate these system requirements $\left(\mathrm{SIR}_{0}, W_{0}\right)$ for the specified $(\rho, B)$.

large, impractical number to use. However, this is not to say that $N$ has to be as large as 19 to support this density. If a greater bandwidth is allocated, the same density can be supported at a lower $N$. For example, allocating $30 \mathrm{kHz}$ of bandwidth allows the system to be designed with $N$ as low as 7 . This system may not be very efficient in terms of bandwidth utilization, because $N=7$ is the capacity-achieving architecture for much lower bandwidths. Nonetheless, it may provide an acceptable practical solution. If the system is constrained to a reuse number

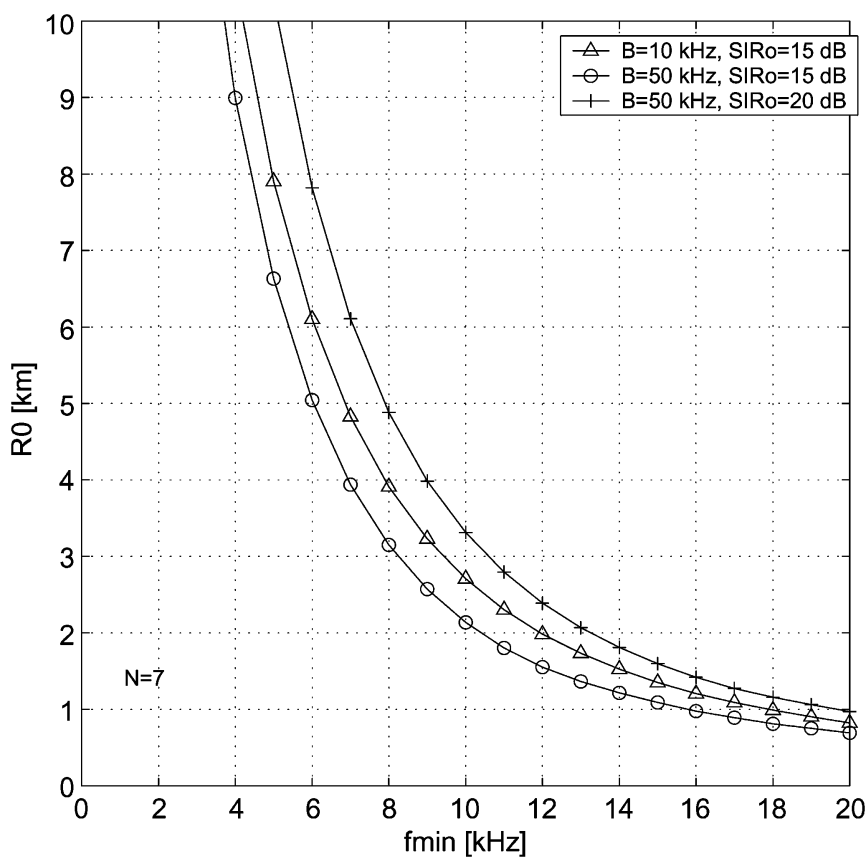

Fig. 10. $R_{0}(N)$ as a function of $f_{\min }$ for several values of $B$ and $\operatorname{SIR}_{0}$.

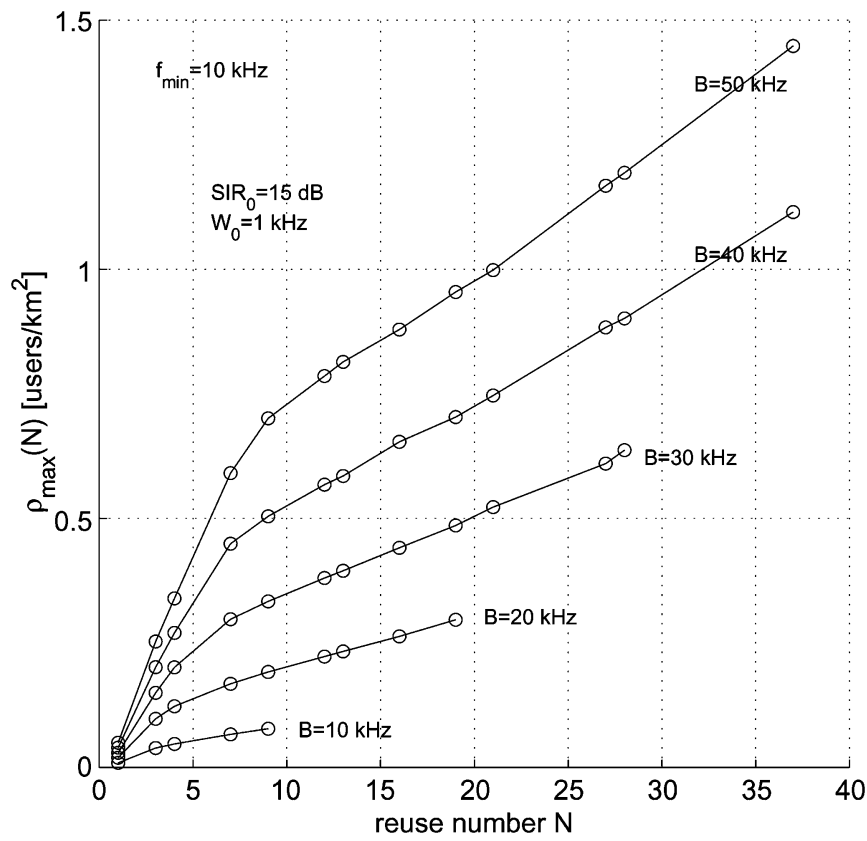

Fig. 11. Maximal user density that can be supported given a fixed reuse number $N$. The system requirements $\left(\operatorname{SIR}_{0}, W_{0}\right)$, the band-edge frequency $f_{\min }$, and several values of the bandwidth $B$ are indicated in this figure.

no greater than 7 , the user density will be limited by the conditional capacity $\rho_{\max }(7)$ (highlighted dashed curve). At $B=$ $50 \mathrm{kHz}$, the capacity curves show that $N=3$ is the lowest $N$ for which $\rho_{\max }(N)>0.25$ users $/ \mathrm{km}^{2}$; hence, the system can be designed with $N$ as low as 3 . This observation is in agreement with the analysis of Section II, where the same parameters $(\rho, B)$ were used in the example of Fig. 7. If the desired user density increases to 0.5 users $/ \mathrm{km}^{2}$, the lowest $N$ that can support it within the same $50 \mathrm{kHz}$ of bandwidth is 7 .

The capacity shown in Fig. 12 corresponds to a predetermined set of system requirements $\left(\mathrm{SIR}_{0}, W_{0}\right)$. For a different set of 


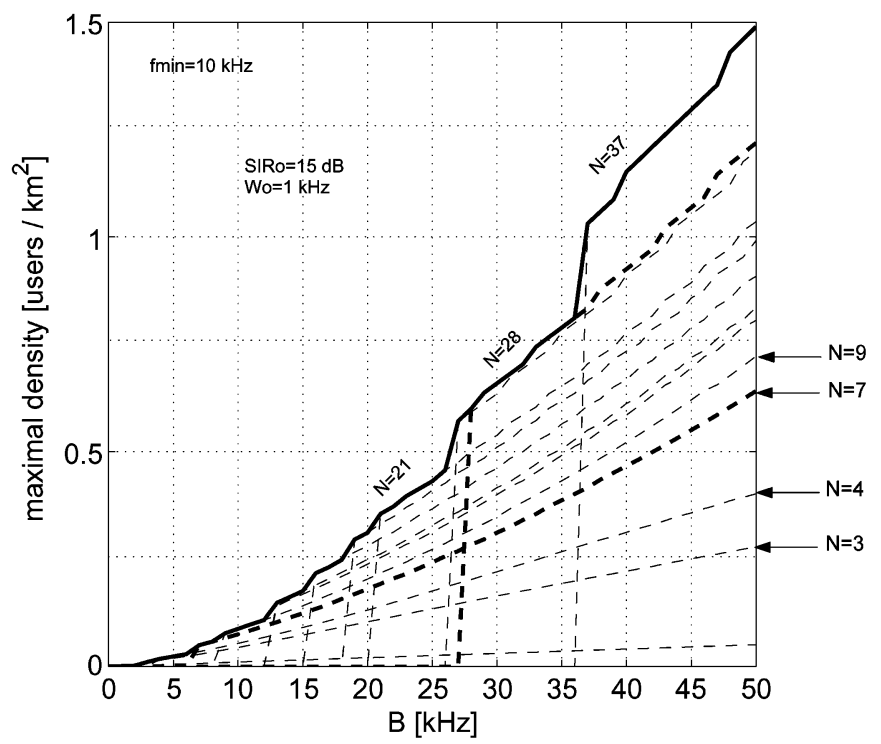

Fig. 12. Capacity of a cellular system: maximal user density $\rho_{\max }$ that can be supported for a given bandwidth $B$, while meeting the system requirements $\left(\mathrm{SIR}_{0}, W_{0}\right)$. Dashed curves represent the conditional capacities $\rho_{\max }(N)$. The band-edge frequency $f_{\min }$ is indicated in this figure.

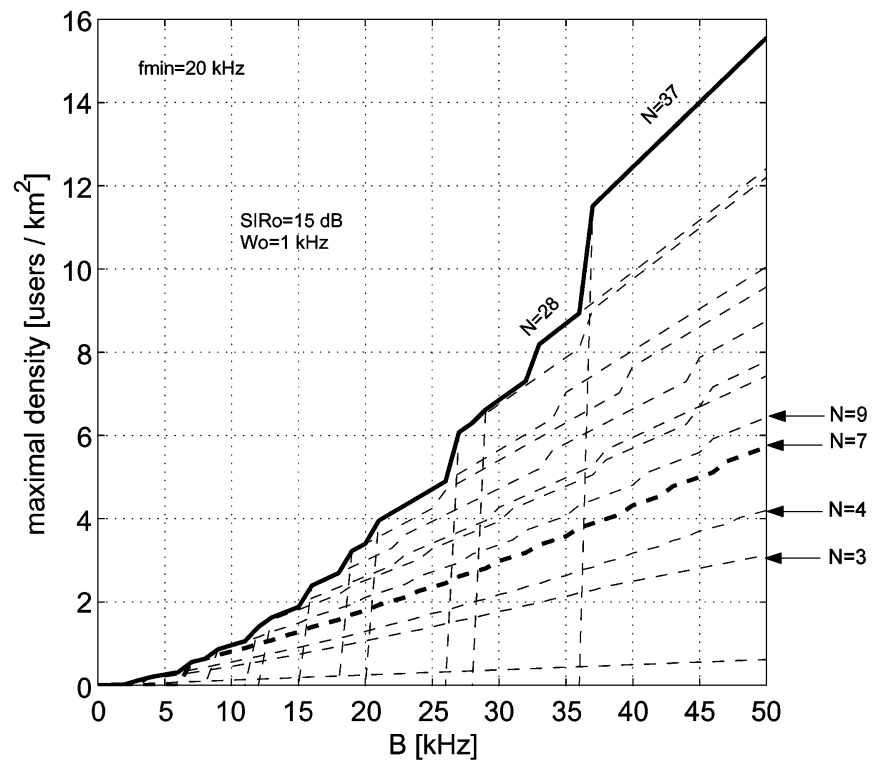

Fig. 13. Capacity of a cellular system: maximal user density $\rho_{\max }$ that can be supported for a given bandwidth $B$, while meeting the system requirements $\left(\operatorname{SIR}_{0}, W_{0}\right)$. Dashed curves represent the conditional capacities $\rho_{\max }(N)$. The band-edge frequency $f_{\min }$ is indicated in this figure.

system requirements, this picture will change. Not surprisingly, imposing stricter requirements causes the capacity to decrease. For example, the capacity drops to less than half of that shown in Fig. 12 for $\operatorname{SIR}_{0}=17 \mathrm{~dB}$ and $W_{0}=1.5 \mathrm{kHz}$ (the system requirements specified in the example of Fig. 9).

In addition to being sensitive to $\left(\operatorname{SIR}_{0}, W_{0}\right)$, the capacity depends on the band-edge frequency $f_{\min }$ [through $R_{0}(N)$ ]. It is particularly interesting to observe the effect of changing $f_{\min }$ on the system capacity. Recall from Fig. 5 that the SIR improves as the operational bandwidth is moved to higher frequencies. This fact bears a significant impact on the system capacity as well. Fig. 13 shows the capacity region for the same system requirements as those of Fig. 12, but a different $f_{\min }$. The total capacity is obviously much greater in this case. In the light of our example, $\rho=0.25$ users $/ \mathrm{km}^{2}$ can now be supported with $B=$ $5 \mathrm{kHz}$ and $N=4$ (which happens to be the capacity-achieving architecture for the requirements specified). The same density can also be supported with $B=7 \mathrm{kHz}$ and a reuse number of 3,4 , or 7 . For a greater density $\rho=1$ user $/ \mathrm{km}^{2}$, the design choices at $B=20 \mathrm{kHz}$ range between $N=3$ and 19 . Note that with a band-edge frequency $f_{\min }=10 \mathrm{kHz}$, it is not possible to support $1 \mathrm{user} / \mathrm{km}^{2}$ within $20 \mathrm{kHz}$ of bandwidth-this $(\rho, B)$ point lies above the capacity limit shown in Fig. 12.

The question that we must inevitably ask at this point is whether the improvement in capacity obtained by moving the operational bandwidth to higher frequencies comes at some price, such as increased transmission power. We address this question in the following section.

\section{POWER AND BANDWIDTh ALLOCATION}

Power and bandwidth allocation, which are already made difficult for a single, interference-free link due to the very nature of frequency-dependent path loss and the ensuing distance-dependent acoustic bandwidth [10], are further complicated in the presence of interference. Selection of $f_{\min }$ is intricately linked with transmission power requirements, which we recall from Section II-A to be responsible for ensuring sufficient SNR and justifying the system design based on SIR.

To illustrate the design choices in power allocation, let us consider an example. Let us assume that the system requirements are specified by $\operatorname{SIR}_{0}=15 \mathrm{~dB}$ and $W_{0}=1 \mathrm{kHz}$, and that the available bandwidth is $B=20 \mathrm{kHz}$. To keep the system practical, we want to use no more than $N=7$. Given a desired user density, say $\rho$ of about 1 users $/ \mathrm{km}^{2}$, the capacity curves $\rho_{\max }(7)$ can be used to find $f_{\min }$ that will meet our requirements. For the example considered, let us use $f_{\min }=20 \mathrm{kHz}$.

Once the frequency $f_{\min }$ has been chosen, we can determine the minimal radius $R_{0}(N)$ that meets the SIR requirement (4). Using the expression (14), we can then choose the cell radius. Let us settle for $R=1 \mathrm{~km}$.

Up to this point, our design was based on the SIR criterion which implicitly assumes that noise is negligible with respect to the signal. This assumption must now be justified through proper selection of the transmission power.

To be precise, let us denote by $\operatorname{SINR}_{n}\left(f_{\text {min }}, P_{T n}\right)$ the SINR for the $n$th cell, which operates using transmission power $P_{T n}$, in the frequency band $\left[f_{n}, f_{n}+B / N\right]$, where $f_{n}=f_{\min }+(n-1)$ $B / N$, and $n=1, \ldots N$. Using the power spectral density $N(f)$ of the ambient noise as in [10], the noise power in the $n$th frequency band is evaluated as

$$
Z_{n}\left(f_{\min }\right)=\int_{f_{n}}^{f_{n}+B / N} N(f) d f .
$$

The corresponding SINR is given by

$$
\operatorname{SINR}_{n}\left(f_{\text {min }}, P_{T n}\right)=\frac{S_{n} R^{-k} I_{n}(R)}{Z_{n}+6 S_{n}(Q R)^{-k} I_{n}(Q R)}
$$

where $S_{n}=P_{T n} /(B / N)$ and $I_{n}(x)$ is computed using (5) with the integration bounds adjusted for the $n$th frequency band. 


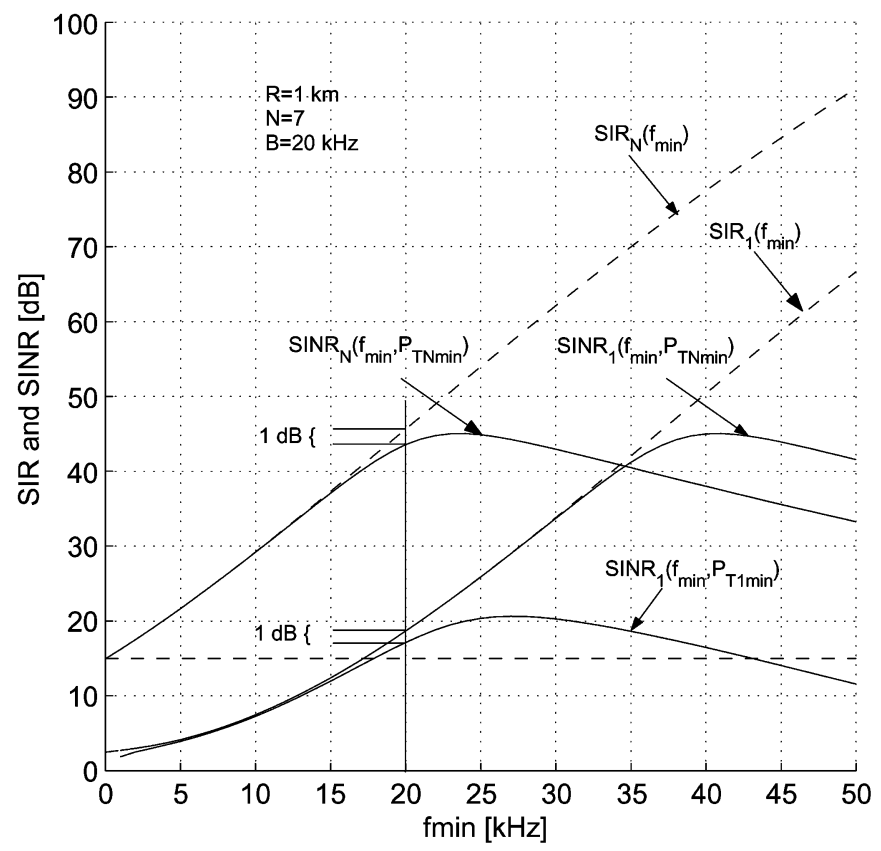

Fig. 14. Determining the minimal transmission power needed to support the SIR-based design.

We now want to find the minimal transmission power $P_{T n \text { min }}$, for which $\mathrm{SINR}_{n} \approx \mathrm{SIR}_{n}, \forall n$. Specifically, let us say that the minimal power is that for which the SINR deviates no more than $1 \mathrm{~dB}$ (or some other level) from the SIR at the chosen frequency $f_{\min }$. To illustrate the process, we will refer to Fig. 14.

When the transmission power is equal for all cells, $P_{T n}=P_{T}$, we have that $\operatorname{SINR}_{n}\left(f_{\text {min }}, P_{T}\right)=\operatorname{SINR}_{1}\left(f_{\text {min }}+\right.$ $\left.(n-1) B / N, P_{T}\right)$. In other words, the SINR curve for the $n$th cell is obtained simply by shifting the SINR curve for the lowest band cell. The same is true for the SIR curves (regardless of the transmission power). The transmission power $P_{T \text { min }}$ must be determined so as to ensure that at $f_{\min }=20 \mathrm{kHz}$, the $1-\mathrm{dB}$ deviation is met for the highest band. The design assumption $\operatorname{SINR}_{n}\left(f_{\text {min }}, P_{T \text { min }}\right) \approx \operatorname{SIR}_{n}\left(f_{\text {min }}\right)$ will then be justified for all the lower bands $n<N$ as well. This fact is illustrated by the curves labeled $\operatorname{SINR}_{N}\left(f_{\min }, P_{T N \text { min }}\right)$ and $\operatorname{SINR}_{1}\left(f_{\min }, P_{T N \min }\right)$. Hence, the minimal power in this case is $P_{T \min }=P_{T N \min }$ (which is evaluated to be $130 \mathrm{~dB}$ re $1 \mu \mathrm{Pa}$ (a $1 \mathrm{~m}$, in this example).

Equal power allocation, however, may not be an efficient choice. In particular, a cell operating in a lower band requires less power to meet the 1-dB SINR deviation rule than does a cell operating in a higher band. Since minimal power is dictated by the highest band in the equal power allocation policy, lower bands are using more power than necessary. The overall system resources are thus wasted. A more efficient power utilization can be achieved if different cells are allowed to use different transmission powers $P_{T n}$. In this case, each cell's transmission power must be determined as the minimum needed to satisfy the $1-\mathrm{dB}$ deviation rule at the chosen $f_{\min }$. Power selection for the lowest frequency band is illustrated by the curve labeled $\operatorname{SINR}_{1}\left(f_{\min }, P_{T 1 \mathrm{~min}}\right)$. The power $P_{T 1 \mathrm{~min}}$ is $30 \mathrm{~dB}$ less than $P_{T N \text { min }}$, the power that would have been consumed by the

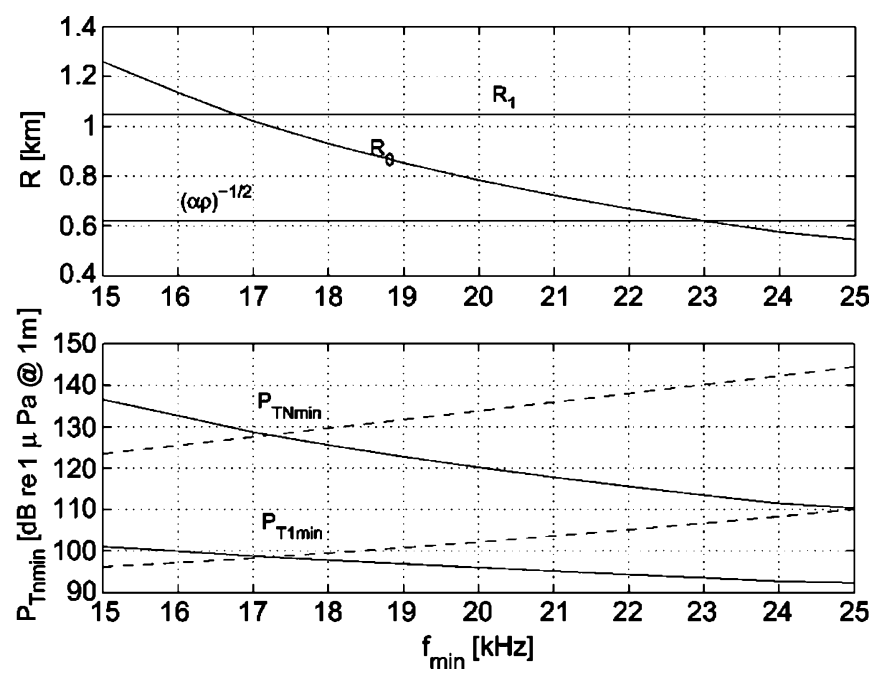

Fig. 15. Cell radius and minimal transmission power as functions of band-edge frequency $f_{\min }$. Solid power curves in the lower plot correspond to cell radius $R=R_{0}$, while dashed curves correspond to fixed cell radius $R=1 \mathrm{~km}$. $\mathrm{SIR}_{0}=15 \mathrm{~dB}, W_{0}=20 \mathrm{kHz}, \rho=1 \mathrm{user} / \mathrm{km}^{2}$, and $N=7$.

lowest band cell under the equal power allocation policy. The overall savings in power obtained through nonuniform allocation are thus considerable.

In summary, Fig. 15 illustrates the minimal power as a function of the band-edge frequency $f_{\text {min }}$. The power is computed as

$$
P_{\text {Tnmin }}=\xi \frac{B}{N} \frac{Z_{n}\left(f_{\min }\right)}{6(Q R)^{-k} I_{n}(Q R)}
$$

where $\xi$ is a scale factor corresponding to the $1-\mathrm{dB}$ deviation.

Two cases are considered in this figure. In the first case, the cell radius is fixed to $R=1 \mathrm{~km}$. The minimal transmission power in this case is shown by the dashed curves for $P_{T 1 \mathrm{~min}}$ and $P_{T N \min }$. Although the SIR improves with increasing $f_{\min }$, the absolute level of the signal at the distance $R$ decreases, requiring more power to make up for the loss in the SNR. Hence, the power needed to ensure the desired SINR $\approx$ SIR increases with $f_{\min }$. In the second case, the cell radius is computed as $R=R_{0}(N)$ for each $f_{\min }$, and this value is used to determine the signal power. The minimal transmission power corresponding to this case is shown in solid curves. It may be somewhat surprising to see that the power now decreases with frequency. This behavior is explained by the simultaneous effect that $f_{\min }$ has on the signal power and on the transmission distance: an increase in frequency requires an increase in transmission power required to span the same distance; however, it also allows a reduction in the cell size. The radius $R_{0}(N)$ is shown in the upper plot as a function of $f_{\min }$ (along with $R_{1}(N)$ and $1 / \sqrt{\alpha \rho}$, which are independent of $f_{\min }$ ). The savings in power that result from transmission over a shorter distance outweigh the expenses required to transmit at higher frequencies, leading to the overall decrease of $P_{T n \min }$ with $f_{\min }$. This fact further speaks in favor of using higher frequency bands.

There is, of course, a limit to increasing the band-edge frequency, not only in that the cell size cannot be shrunk indefinitely, but also from the practical viewpoint of transducer design and adjacent channel interference. However, moving the operational bandwidth to higher frequencies within the practical 
system limitations yields a greater user capacity, and reduces the overall power consumption provided that the cell radius is kept at a minimum.

\section{CONCLUSION}

The concept of an underwater acoustic cellular network was considered as one that may be of interest to the future systems of distributed autonomous nodes, where spatial frequency reuse may enable large coverage within the constraints of limited acoustic bandwidth. The system design and capacity analysis presented in this paper take into account the physical laws of sound propagation, revealing interesting conclusions that emphasize the fact that existing solutions from the terrestrial radio systems do not always apply to the underwater acoustic environment.

The basic acoustic propagation loss leads to a set of constraints that the cell radius and the reuse pattern $(R, N)$ must satisfy in order to constitute an admissible solution for the cellular system topology. In particular, for the given user density and the system bandwidth $(\rho, B)$, and the quality constraints $\left(\mathrm{SIR}_{0}, W_{0}\right)$ on the SIR and the bandwidth per user, only those values of $(R, N)$ that belong to a certain region provide a solution to the system design. The region of admissible $(R, N)$-if existent-defines the possible network topologies. This result is in stark contrast to the land-based radio systems, where $\operatorname{SIR}_{0}$ alone determines the reuse number, bearing no influence on the cell radius.

The fact that the range of admissible cell radii is bounded implies a limit on the overall system capacity, which we defined as the maximal density of users $\rho_{\max }$ that can be supported within a given bandwidth. Analytical results show that capacity-achieving architectures are characterized by the reuse number $N$ that grows with $\rho_{\max }$. Numerical examples were used to illustrate how the capacity region and the associated capacity-achieving architectures depend on the system constraints $\left(\mathrm{SIR}_{0}, W_{0}\right)$, as well as on the band-edge frequency $f_{\text {min }}$. When the operational frequency band is centered at lower frequencies, reuse numbers (much) greater than 7 may be needed to support a desired density of users without expanding the bandwidth beyond the minimum needed. Nonetheless, if bandwidth is available, it can be traded off for a lower, more practical reuse number. Alternatively, moving the operational frequency band to higher frequencies greatly improves the system capacity by virtue of increasing the SIR. It also requires less transmission power, provided that the cell radius is kept at a minimum. Total power consumption can further be reduced through nonuniform power allocation across the cells.

The apparent constraints involved in the system design, and their impact on the user capacity, serve as a motivation for further research into the spatial reuse methods that will enable effective interference mitigation while remaining efficient in terms of both bandwidth and power usage. For example, such methods may capitalize on the low speed of sound underwater [14]. While acoustic propagation dictates complex relationships between the various system parameters, the analysis presented can easily be applied to any set of system constraints. It thus offers a simple tool that can be used to obtain basic guidelines for the design of future underwater networks based on the cellular types of architecture.

\section{REFERENCES}

[1] J. Rice, "SeaWeb acoustic communication and navigation networks," in Proc. Int. Conf. Underwater Acoust. Meas.: Technol. Results, Crete, Greece, Jun. 2005, pp. 2007-2017.

[2] M. Grund, J. Preisig, L. Freitag, and K. Ball, "The PLUSNet underwater communications system: Acoustic telemetry for undersea surveillance," in Proc. IEEE OCEANS Conf., Boston, MA, Sep. 2006, DOI: 10.1109/OCEANS.2006.307036.

[3] M. Stojanovic and L. Freitag, "Multichannel detection for wideband underwater acoustic CDMA communications," IEEE J. Ocean. Eng., vol. 31, no. 3, pp. 685-695, Jul. 2006.

[4] F. Salva-Garau and M. Stojanovic, "Multi-cluster protocol for ad hoc mobile underwater acoustic networks," in Proc. IEEE OCEANS Conf., San Diego, CA, Sep. 2003, vol. 1, pp. 91-98.

[5] M. Molins and M. Stojanovic, "Slotted FAMA: A MAC protocol for underwater acoustic networks," in Proc. IEEE OCEANS Conf., Singapore, May 2006, DOI: 10.1109/OCEANSAP.2006.4393832.

[6] X. Guo, M. Frater, and M. Ryan, "A propagation-delay-tolerant collision avoidance protocol for underwater acoustic sensor networks," in Proc. IEEE OCEANS Conf., Singapore, 2006, DOI: 10.1109/OCEANSAP.2006.4393849.

[7] B. Peleato and M. Stojanovic, "Distance aware collision avoidance protocol for ad-hoc underwater acoustic sensor networks," IEEE Commun. Letters, vol. 11, no. 12, pp. 1025-1027, Dec. 2007.

[8] V. Rodoplu and M. Park, "An energy-efficient MAC protocol for wireless acoustic networks," in Proc. IEEE OCEANS Conf., Washington, DC, Sep. 2005, vol. 2, pp. 710-720.

[9] W. Ye, J. Heidemann, and D. Estrin, "Medium access control with coordinated adaptive sleeping for wireless sensor networks," IEEE/ACM Trans. Netw., vol. 12, no. 3, pp. 493-506, Jun. 2004

[10] M. Stojanovic, "On the relationship between capacity and distance in an underwater acoustic channel," in Proc. 1st ACM Int. Workshop Underwater Netw., Los Angeles, CA, Sep. 2006, pp. 41-47.

[11] J. Partan, J. Kurose, and B. N. Levine, "A survey of practical issues in underwater networks," in Proc. 1st ACM Int. Workshop Underwater Netw., Los Angeles, CA, Sep. 2006, pp. 17-24.

[12] T. S. Rappaport, Wireless Communications: Principles and Practice. Englewood Cliffs, NJ: Prentice-Hall, 1996.

[13] L. Berkhovskikh and Y. Lysanov, Fundamentals of Ocean Acoustics. New York: Springer-Verlag, 1982.

[14] B. Peleato and M. Stojanovic, "A channel sharing scheme for underwater cellular networks," in Proc. IEEE OCEANS Eur. Conf., 2007, DOI: 10.1109/OCEANSE.2007.4302232.

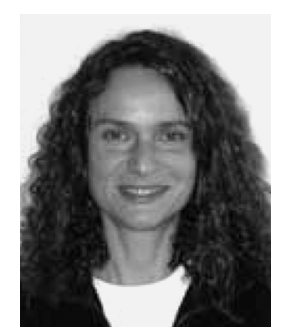

Milica Stojanovic graduated from the University of Belgrade, Belgrade, Serbia, in 1988, and received the M.S. and Ph.D. degrees in electrical engineering from Northeastern University, Boston, MA, in 1991 and 1993, respectively.

Currently, she is a Principal Scientist at the Massachusetts Institute of Technology, Cambridge, and also a Guest Investigator at the Woods Hole Oceanographic Institution, Woods Hole, MA. Her research interests include digital communications theory and statistical signal processing, and their applications to mobile radio and underwater acoustic communication systems. 